\title{
Bifurcations in the Kuramoto model on graphs
}

\author{
Hayato Chiba, ${ }^{*}$ Georgi S. Medvedev ${ }^{\dagger}$ and Matthew S. Mizuhara ${ }^{\ddagger}$
}

May 11, 2018

\begin{abstract}
In his classical work, Kuramoto analytically described the onset of synchronization in all-to-all coupled networks of phase oscillators with random intrinsic frequencies. Specifically, he identified a critical value of the coupling strength, at which the incoherent state loses stability and a gradual build-up of coherence begins. Recently, Kuramoto's scenario was shown to hold for a large class of coupled systems on convergent families of deterministic and random graphs [4, 5]. Guided by these results, in the present work, we study several model problems illustrating the link between network topology and synchronization in coupled dynamical systems.

First, we identify several families of graphs, for which the transition to synchronization in the Kuramoto model starts at the same critical value of the coupling strength and proceeds in practically the same way. These examples include Erdős-Rényi random graphs, Paley graphs, complete bipartite graphs, and certain stochastic block graphs. These examples illustrate that some rather simple structural properties such as the volume of the graph may determine the onset of synchronization, while finer structural features may affect only higher order statistics of the transition to synchronization. Further, we study the transition to synchronization in the Kuramoto model on power law and small-world random graphs. The former family of graphs endows the Kuramoto model with very good synchronizability: the synchronization threshold can be made arbitrarily low by varying the parameter of the power law degree distribution. For the Kuramoto model on small-world graphs, in addition to the transition to synchronization, we identify a new bifurcation leading to stable random twisted states. The examples analyzed in this work complement the results in [4, 5].
\end{abstract}

\section{Introduction}

The Kuramoto model of coupled phase oscillators provides an important paradigm for studying collective dynamics and synchronization in ensembles of interacting dynamical systems. In its original form, the KM describes the dynamics of all-to-all coupled phase oscillators with randomly distributed intrinsic frequencies

$$
\dot{\theta}_{i}=\omega_{i}+K n^{-1} \sum_{j=1}^{n} \sin \left(\theta_{j}-\theta_{i}\right), \quad i \in[n]:=\{1,2, \ldots, n\} .
$$

\footnotetext{
*Institute of Mathematics for Industry, Kyushu University / JST PRESTO, Fukuoka, 819-0395, Japan, chibalimi.kyushu-u.ac.jp

${ }^{\dagger}$ Department of Mathematics, Drexel University, 3141 Chestnut Street, Philadelphia, PA 19104, medvedev@drexel. edu

${ }^{\ddagger}$ Department of Mathematics and Statistics, The College of New Jersey, mi zuharmetcnj . edu
} 
Here, $\theta_{i}: \mathbb{R} \rightarrow \mathbb{T}:=\mathbb{R} / 2 \pi \mathbb{Z}$ is the phase of the oscillator $i \in[n]$. The intrinsic frequencies $\omega_{i}, i \in[n]$, are independent identically distributed random variables. The density of the probability distribution of $\omega_{1}$, $g$, is a smooth even function. The sum on the right-hand side of (1.1) models the interactions between the oscillator $i$ and the rest of the network. Parameter $K$ controls the strength of the interactions. The sign of $K$ determines the type of interactions. The coupling is attractive if $K>0$, and is repulsive otherwise. Sufficiently strong attractive coupling favors synchrony.

For small positive values of $K$, the KM shows little coherence. The phases fill out the unit circle approximately uniformly (Fig. 1 1 ). This dynamical regime is called an incoherent state. It persists for positive values of $K$ smaller than the critical value $K_{c}=2(\pi g(0))^{-1}$. For values of $K>K_{c}$, the system undergoes a gradual build-up of coherence approaching complete synchronization as $K \rightarrow \infty$ (Fig. 1 $1 \mathrm{~b}$ ). Kuramoto identified the critical value $K_{c}$ and described the transition to synchrony, using the complex order parameter

$$
h(t)=n^{-1} \sum_{j=1}^{n} e^{i \theta_{j}(t)} .
$$

Below, we will often use the polar form of the order parameter

$$
h(t)=r(t) e^{i \psi(t)} .
$$

Specifically, he showed that for $t \gg 1$ (cf. [19])

$$
r(t)= \begin{cases}O\left(n^{-1 / 2}\right), & 0<K<K_{c} \\ r_{\infty}\left(K-K_{c}\right)+O\left(n^{-1 / 2}\right), & K>K_{c}\end{cases}
$$

where

$$
r_{\infty}(x)=r_{1} \sqrt{x}+O(x), x \geq 0, \quad r_{1}=\frac{4}{K_{c}^{2} \sqrt{-\pi g^{\prime \prime}(0)}} .
$$

Here, $0 \leq r(t) \leq 1$ and $\psi(t)$ stand for the modulus and the argument of the order parameter defined by the right-hand side of (1.2). The value of $r$ is interpreted as a measure of coherence in the system dynamics. Indeed, if all phases are independent random variables distributed uniformly on $\mathbb{T}$ (complete incoherence) then $r=o(1)$ with probability one, by the Strong Law of Large Numbers. If, on the other hand, all phase variables are equal then $r=1$. Equations (1.4), (1.5) suggest that the system undergoes a pitchfork bifurcation en route to synchronization. This bifurcation is clearly seen in the numerical experiments (see Figure 11). Furthermore, the Kuramoto's scenario of the transition to synchrony was recently confirmed by rigorous mathematical analysis of the KM [3, 6, 7].

In this paper, we investigate bifurcations in the $\mathrm{KM}$ on a variety of graphs ranging from symmetric Caley graphs to random small-world and power law graphs. To visualize the connectivity of a large graph, we use the pixel picture of a graph. This is a square black and white plot, where each black pixel stands for entry 1 in the adjacency matrix of the graph (see Figure 2). As we show below, the graph structure plays a role in the transition to synchrony. Furthermore, some graphs may force bifurcations to spatial patterns other than synchrony. To highlight the role of the network topology, we present numerical experiments of the KM on small-world graphs [20]. These graphs are formed by replacing some of the edges of a regular graph with random edges (see Figure $2 \mathbf{a}$ ). These results demonstrate that some graphs may exhibit bifurcations to spatial patterns other than synchrony. 


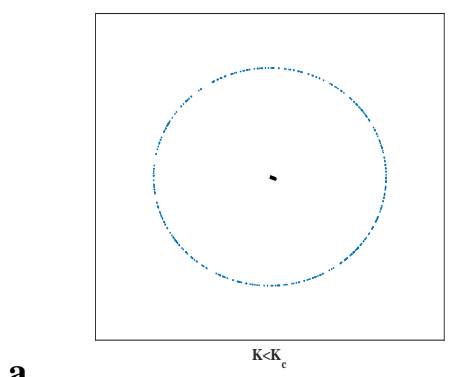

b
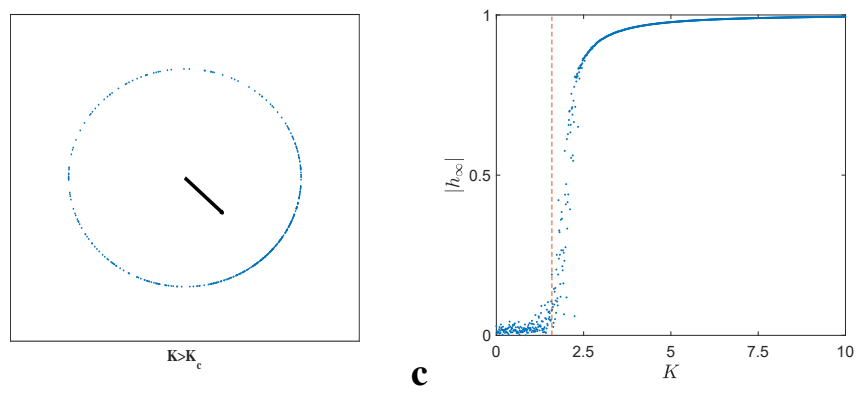

Figure 1: The distribution of the phases of coupled oscillators is shown on the unit circle in the complex plane: $\theta_{k} \mapsto e^{i \theta_{k}} \in \mathbb{C}, k \in[n]$. The strength of coupling is below the critical value $K_{c}$ in (a) and is above $K_{c}$ in (b). The black arrow depicts the order parameter, as a vector in the complex plane (cf. (1.2)). The bigger size (modulus) of the order parameter corresponds to the higher degree of coherence. In (a) the modulus of the order parameter is close to zero, and the distribution of the oscillators is close to the uniform distribution. In contrast in (b), the distribution develops a region of higher density. The complex order parameter points to the expected value (center of mass) of the distribution of the oscillators around $\mathbb{T}$. c) The modulus of the order parameter is plotted for different values of $K$. The gradual change of the modulus of the order parameter from values around zero to those close to 1 marks the transition to synchronization.

Figure 3 shows that for the KM on small-world graphs like in that on complete graphs, the order parameter undergoes a smooth transition 1 . Near the critical value $K_{c}^{+} \approx 3.2$, the asymptotic in time value of the order parameter starts to grow monotonically and approaches 0.5 for increasing values of $K$. This change in the order parameter corresponds to the transition to synchronization. Interestingly, in contrast to the original KM, the model on small-world graphs exhibits another transition at a negative value of $K$, $K_{c} \approx-27$ (Figure 3b). This time it grows monotonically for decreasing values of $K$ and approaches the value approximately equal to 0.0475 . The corresponding state of the network is shown in Figure 4 a. In the pattern shown in this figure, the oscillators are distributed randomly about a 2-twisted state. A $q$-twisted state $(q \in \mathbb{Z})$ is a linear function on the unit circle: $T_{q}(x)=\frac{2 \pi q x}{n} \bmod 2 \pi, x \in[n], q$-twisted states have been studied before as stable steady states in repulsively coupled KM on Caley graphs [17, 21]. By changing parameters controlling small-world connectivity, we find different $q$-twisted states bifurcating from the incoherent state for decreasing negative $K$ (see Figure $4 \mathbf{a}, \mathbf{b}$ ). These numerical experiments show that in the $\mathrm{KM}$ on small-world graphs the incoherent state is stable for $K \in\left(K_{c}^{-}, K_{c}^{+}\right)$. For values $K>K_{c}^{+}$, the system undergoes the transition to synchrony, while for $K<K_{c}^{-}$, it leads to random patterns localized around $q$-twisted states.

The loss of stability of the incoherent state in the KM on spatially structured networks was analyzed in [4, 5]. The linear stability analysis of the incoherent state yields the critical values $K_{c}^{ \pm}$[4], and the analysis of the bifurcations at $K_{c}^{ \pm}$explains the emerging spatial patterns [5]. In the present work, using the insights from [4, 5], we conduct numerical experiments illustrating the role of the network topology in shaping the transition from the incoherent state to coherent structures like synchronous and twisted states in the KM on graphs. In particular, we present numerical simulations showing for many distinct graphs the onset of synchronization takes place at the same critical value and the graph structure has only higher order effects.

\footnotetext{
${ }^{1}$ For the KM on graphs, the order parameter must be suitably redefined (see $(3.7)$ ).
} 


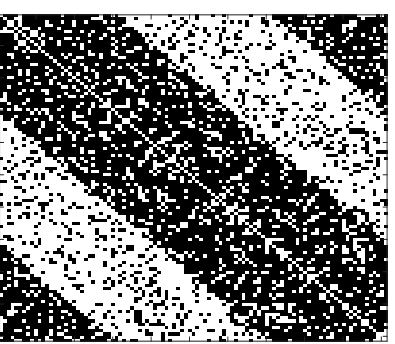

a b

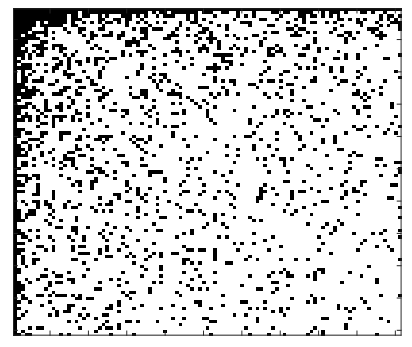

Figure 2: Pixel pictures of small-world (a) and power law (b) graphs on 100 nodes.
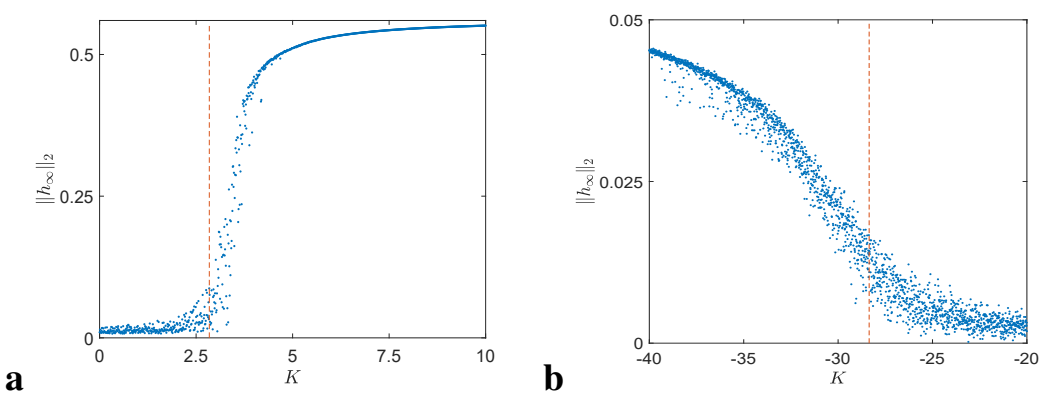

Figure 3: Transition to synchronization in the KM on small-world graphs (a). For negative $K$, the KM undergoes another transition at $K_{c}^{-}<0$ (b). The emerging pattern is shown in Figure 4 a,b.

We identify the dominant structural properties of the graphs shaping the transition to synchronization in the KM.

The effective analysis of the KM on large graphs requires an analytically convenient description of graph sequences. To this end, we employ the approach developed in [14, 15] inspired by the theory of graph limits [11] and, in particular, by W-random graphs [12]. We explain the graph models used in this paper in Section 2. The W-random graph framework affords an analytically tractable mean field description of the KM on a broad class of dense and sparse graphs. The mean field partial differential equation for the $\mathrm{KM}$ on graphs is explained in Section 3 . There we also explain the generalization of the order parameter suitable for the KM on graphs. After that we go over the main results of [4, 5]. In Section 4, we present our numerical results. We use carefully designed examples of graphs to highlight the structural properties affecting the onset of synchrony. For instance, we show that the KMs on Erdős-Renyi and Paley graphs have the same critical value $K_{c}$ marking the onset of synchronization. Furthermore, the mean field limits of the $\mathrm{KM}$ on these graphs coincide with that for the KM on weighted complete graph. We demonstrate the similarity in the transition to synchronization in the KM on bipartite graphs and on the family of stochastic block graphs interpolating a disconnected graph and a weighted complete graph. This time the mean field equations for the two models are different, but the critical value $K_{c}$ remains the same. Further, we investigate the bifurcations in the KM on small-world and power law graphs illuminating the effects of these random connectivity patterns on the dynamics of the KM. 

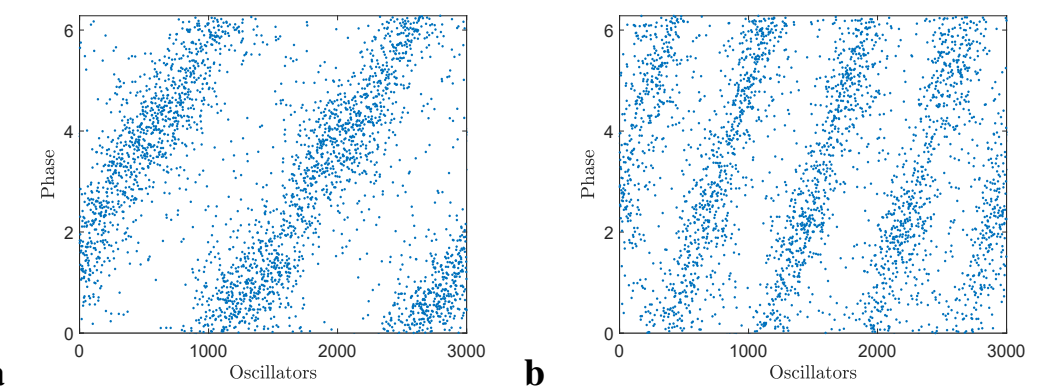

Figure 4: The oscillators in a are clustered around a 2-twisted state $(p=.2, r=.3, K=-36$; see Example 2.1 for the definitions of parameters). By changing one of the parameters controlling small-world connectivity (namely, by reducing the range of local interactions), one can obtain $q$-twisted states for different values of $q \in \mathbb{Z}$, such as the 4 -twisted state shown in plot $\mathbf{b}(p=.2, r=.2, K=-40)$.

\section{The KM on graphs}

We begin with the description of graph models that will be used in this paper. Let $\Gamma_{n}=\left\langle V\left(\Gamma_{n}\right), E\left(\Gamma_{n}\right), A_{n}\right\rangle$ be a weighted directed graph on $n$ nodes. $V\left(\Gamma_{n}\right)=[n]$ stands for the node set of $\Gamma_{n}$. $A_{n}=\left(a_{n, i j}\right)$ is an $n \times n$ weight matrix. The edge set

$$
E\left(\Gamma_{n}\right)=\left\{\{i, j\} \in[n]^{2}: a_{n, i j} \neq 0\right\} .
$$

If $\Gamma_{n}$ is a simple graph then $A_{n}$ is the adjacency matrix.

The KM on $\Gamma_{n}$ is defined as follows

$$
\begin{aligned}
\dot{u}_{n, i} & =\omega_{i}+K\left(n \alpha_{n}\right)^{-1} \sum_{j=1}^{n} a_{n, i j} \sin \left(u_{n, j}-u_{n, i}\right), \quad i \in[n], \\
u_{n, i}(0) & =u_{n, i}^{0} .
\end{aligned}
$$

The sequence $\alpha_{n} \neq 0$ is only needed if $\left\{\Gamma_{n}\right\}$ is a sequence of sparse graphs. Without proper rescaling, the continuum limit (as $n \rightarrow \infty$ ) of the KM on sparse graphs is trivial. The sequence $\left\{\alpha_{n}\right\}$ will be explained below, when we introduce sparse $W$-random graphs. Until then one can can assume $\alpha_{n} \equiv 1$.

We will be studying solutions of 2.1 in the limit as $n \rightarrow \infty$. Clearly, such limiting behavior is possible only if the sequence of graphs $\left\{\Gamma_{n}\right\}$ is convergent in the appropriate sense. We want to define $\left\{\Gamma_{n}\right\}$ in such a way that it includes graphs of interest in applications, and at the same time is convenient for deriving the continuum limit. To achieve this goal, we use the ideas from the theory of graph limits [11]. Specifically, we choose a square integrable function $W$ on a unit square $I^{2}:=[0,1]^{2} . W$ is used to define the asymptotic behavior of $\left\{\Gamma_{n}\right\}$ as $n \rightarrow \infty$. It is called a graphon in the theory of graph limits [11]. Let

$$
\mathcal{W}:=\left\{W \in L^{2}\left(I^{2}\right): \quad W(x, y)=W(y, x) \text { a.e. }\right\} .
$$

We define $\mathcal{W}_{0} \subset \mathcal{W}$ as a set of measurable symmetric functions on $I^{2}$ with values in $I$. We discretize the unit interval $I: x_{n, j}=j / n, j \in\{0\} \cup[n]$ and denote $I_{n, i}:=\left(x_{n, i-1}, x_{n, i}\right], i \in[n]$. 
The following graph models are inspired by W-random graphs [12, 2]. Let $\Gamma_{n}=\left\langle V\left(\Gamma_{n}\right), E\left(\Gamma_{n}\right), A_{n}=\right.$ $\left.\left(a_{n, i j}\right)\right\rangle$.

(DD) Weighted deterministic graph $\Gamma_{n}=H(n, W)$ is defined as follows:

$$
a_{n, i j}=\langle W\rangle_{I_{n, i} \times I_{n, j}}:=n^{2} \int_{I_{n, i} \times I_{n, j}} W(x, y) d x d y
$$

where $W \in \mathcal{W}$.

(RD) Dense random graph $\Gamma_{n}=H_{r}(n, W)$. Let $W \in \mathcal{W}_{0}$ and $a_{n, i j}, 1 \leq i \leq j \leq n$, be independent identically distributed (IID) random variables such that

$$
\mathbb{P}\left(a_{n, i j}=1\right)=\langle W\rangle_{I_{n, i} \times I_{n, j}}, \quad \mathbb{P}\left(a_{n, i j}=0\right)=1-\mathbb{P}\left(a_{n, i j}=1\right) .
$$

(RS) Sparse random graphs $\Gamma_{n}=H_{r}\left(n, W,\left\{\alpha_{n}\right\}\right)$. Let $W \in \mathcal{W}$ be a nonnegative function and positive sequence $1 \geq \alpha_{n} \searrow 0$ satisfy $n \alpha_{n} \rightarrow \infty$ as $n \rightarrow \infty$,

$$
\mathbb{P}\left(\{j, i\} \in E\left(\Gamma_{n}\right)\right)=\alpha_{n}\left\langle\tilde{W}_{n}\right\rangle_{I_{n, i} \times I_{n, j}}, \quad \tilde{W}_{n}(x, y):=\min \left\{\alpha_{n}^{-1}, W(x, y)\right\} .
$$

We illustrate the random graph models in (RD) and (RS) with the following examples.

Example 2.1. (SW) Small-world graph $S_{n, r, p}=H_{r}\left(n, W_{r, p}\right), r \in(0,0.5), p \in(0,0.5]$, is defined via

$$
W_{p, r}(x, y)= \begin{cases}1-p, & \min \{|x-y|, 1-|x-y|\} \leq r \\ p, & \text { otherwise }\end{cases}
$$

(see Figure 2a).

(ER) Erdös-Rényi graph $G_{n, p}=H_{r}\left(n, W_{p}\right), W_{p} \equiv p \in(0,1)$.

(SER) Sparse Erdös-Rényi graph $\tilde{G}_{n, p}=H_{r}\left(n, W_{p},\left\{n^{-\beta}\right\}\right), \quad \beta \in(0,1)$.

(PL) Power law graph $\Gamma=H_{r}\left(n, W^{\gamma},\left\{n^{-\beta}\right\}\right)$

$$
W^{\gamma}(x, y)=(x y)^{-\gamma}, \quad 0<\gamma<\beta<1,
$$

(see Figure $2 \boldsymbol{b}$ ).

Graphon $W$ carries all information needed for the derivation of the continuum limit for the $\mathrm{KM}$ on deterministic and random graphs (DD), (RD), and (RS). However, for the KM on random graphs (RD) and (RS) taking the continuum limit involves an additional step. Namely, we first approximate the model on random graph by that on the averaged deterministic weighted graph $\Gamma_{n}=H(n, W)$ :

$$
\begin{aligned}
\dot{v}_{n, i} & =\omega_{n, i}+K n^{-1} \sum_{j=1}^{n} \bar{W}_{n, i j} \sin \left(v_{n, j}-v_{n, i}\right), \quad i \in[n], \\
v_{n, i}(0) & =u_{n, i}^{0},
\end{aligned}
$$

where $\bar{W}_{n, i j}:=\left\langle\tilde{W}_{n}\right\rangle_{I_{n, i} \times I_{n, j}}$.

The approximate model (2.1) is formally derived from the original $\mathrm{KM} 2.1$ on a random graph $\Gamma_{n}=$ $H_{r}\left(n, W,\left\{\alpha_{n}\right\}\right)$ by averaging the right hand side of 2.1) over all possible realizations of $\Gamma_{n}$. The justification of averaging is given in [8]. 


\section{The mean field limit}

Solutions of the KM with distributed frequencies such as incoherent state and the solutions bifurcating from the incoherent state are best described in statistical terms. To this end, suppose $\rho(t, u, \omega, x)$ is the conditional density of the random vector $(u, \omega)$ given $\omega$, and parametrized by $(t, x) \in \mathbb{R}^{+} \times I$. Here, the spatial domain $I=[0,1]$ represents the continuum of the oscillators in the limit $n \rightarrow \infty$ (see [4] for precise meaning of the continuum limit). Then $\rho$ satisfies the following initial value problem

$$
\begin{aligned}
\frac{\partial}{\partial t} \rho(t, u, \omega, x)+\frac{\partial}{\partial u}\{\rho(t, u, \omega, x) V(t, u, \omega, x)\} & =0 \\
\rho(0, u, \omega, x) & =\rho^{0}(u),
\end{aligned}
$$

where initial density $\rho^{0}(u)$ independent of $\omega$ and $x$ for simplicity (see [8] for the treatment of a more general case). The velocity field $V$ is defined via

$$
V(t, u, \omega, x)=\omega+K \int_{I} \int_{\mathbb{R}} \int_{\mathbb{T}} W(x, y) \sin (\phi-u) \rho(t, \phi, \omega, y) g(\omega) d \phi d \omega d y .
$$

The density $\rho(t, \cdot)$ approximates the distribution of the oscillators around $\mathbb{T}$ at time $t \in[0, T]$. Specifically, in the large $n$ limit the empirical measures defined on the Borel $\sigma$-algebra $\mathcal{B}(G), G:=\mathbb{T} \times \mathbb{R} \times I$ :

$$
\mu_{t}^{n}(A)=n^{-1} \sum_{i=1}^{n} \delta_{\left(u_{n i}(t), \omega_{i}, x_{n i}\right)}(A), \quad A \in \mathcal{B}(G),
$$

converge weakly to the absolutely continuous measure

$$
\mu_{t}(A)=\int_{A} \rho(t, u, \omega, x) g(\omega) d u d \omega d x
$$

provided the initial data (2.2) converge weakly to $\mu_{0}$ (cf. [4, Theorem 2.2], see also [8]).

The mean field limit provides a powerful tool for studying the KM. It gives a simple analytically tractable description of complex dynamics in this model. In particular, the incoherent state in the mean field description corresponds to the stationary density $\rho_{u}=1 / 2 \pi$. The stability analysis of $\rho_{u}$, a steady state solution of (3.1), yields the region of stability of the incoherent state and the critical values of $K$, at which it loses stability. Furthermore, the analysis of the bifurcations at the critical values of $K$ explains the phase transitions in the $\mathrm{KM}$ (cf. [5]). Importantly, we can trace the role of the network topology in the loss of stability of the incoherent state and emerging spatial patterns. In the remainder of this section, we informally review some of the results of [4, 5], which will be used below.

The key ingredient in the analysis of the KM on graphs, which was not used in the analysis of the original $\mathrm{KM}$, is the the following kernel operator $\mathbf{W}: L^{2}(I) \rightarrow L^{2}(I)$

$$
\mathbf{W}[f](x)=\int_{\mathbb{R}} W(x, y) f(y) d y .
$$

Recall that $W \in L^{2}\left(I^{2}\right)$ is a symmetric function. Thus, $\mathbf{W}: L^{2}\left(I^{2}\right) \rightarrow L^{2}\left(I^{2}\right)$ is a self-adjoint compact operator. The eigenvalues of $\mathbf{W}$, on the one hand, carry the information about the structure of the graphs 
in the sequence $\left\{\Gamma_{n}\right\}$, on the other hand, they appear in the stability analysis of the incoherent state. Thus, through the eigenvalues of $\mathbf{W}$ we can trace the relation between the structure of the network and the onset of synchronization in the KM on graphs.

Since $\mathbf{W}$ is self-adjoint and compact, the eigenvalues of $\mathbf{W}$ are real and bounded with the only possible accumulation point at 0 . In all examples considered in this paper, the largest eigenvalue $\mu_{\max }>0$ and the smallest $\mu_{\min } \leq 0$. The linear stability analysis in [4] shows that the incoherent state is stable for $K \in\left[K_{c}^{-}, K_{c}^{+}\right]$, where

$$
K_{c}^{-}=\frac{2}{\pi g(0) \mu_{\min }} \quad \text { and } \quad K_{c}^{+}=\frac{2}{\pi g(0) \mu_{\max }} .
$$

Except for small-world graphs, for all other graphs considered below, the smallest eigenvalue $\mu_{\min }=0$. Thus, the incoherent state in the $\mathrm{KM}$ on these graphs is stable for $K \leq K_{c}^{+}$. In this section, we comment on the bifurcation at $K_{c}^{+}$and postpone the discussion of the bifurcation at $K_{c}^{-}$until Section 5, where we deal with the KM on small-world graphs.

The analysis of the bifurcations in the KM on graphs relies on the appropriate generalization of the Kuramoto's order parameter (cf. [4])

$$
h_{n}(t)=\left(h_{n 1}(t), h_{n 2}(t), \ldots, h_{n n}(t)\right), \quad h_{n i}(t)=\frac{1}{n} \sum_{j=1}^{n} W_{n, i j} e^{i \theta_{n j}(t)}, i \in[n],
$$

and its continuous counterpart

$$
h(t, x)=\int_{I} \int_{\mathbb{R}} \int_{\mathbb{T}} W(x, y) e^{i \theta} \rho(t, \theta, \omega, y) g(\omega) d \theta d \omega d y .
$$

The value of the continuous order parameter 3.8$) h(t, x)$ carries the information about the (local) degree of coherence at point $x$ and time $t$. It is adapted to a given network connectivity through the kernel $W$.

The main challenge in the stability analysis of the incoherent state lies in the fact that for $K \in\left[K_{c}^{-}, K_{c}^{+}\right]$ the linearized operator has continuous spectrum on the imaginary axis and no eigenvalues (cf. [4]). To overcome this difficulty, in [5], the generalized spectral theory was used to identify generalized eigenvalues responsible for the instability at $K_{c}^{+}$, and to construct the finite-dimensional center manifold. The explanation of these results in beyond the scope of this paper. An interested reader is referred to [5] for details. Here, we only present the main outcome of the bifurcation analysis. The center manifold reduction for the order parameter near $K_{c}^{+}$yields a stable branch of solutions:

$$
h_{\infty}(x, K)=\frac{g(0)^{2} \pi^{3 / 2}}{\sqrt{-g^{\prime \prime}(0)}} \mu_{\max }^{3 / 2} C(x) \sqrt{K-K_{c}^{+}}+o\left(\sqrt{K-K_{c}^{+}}\right), \quad 0<K-K_{c}^{+} \ll 1,
$$

where $C(x)$ is defined through the eigenfunction of $\mathbf{W}$ corresponding to $\mu_{\max }$ (see [5] for details). Formula (3.9) generalizes the classical Kuramoto's formula describing the pitchfork bifurcation in the all-to-all coupled model to the KM on graphs. The network structure enters into the description of the pitchfork bifurcation through the largest eigenvalue $\mu_{\max }$ and the corresponding eigenspace. 


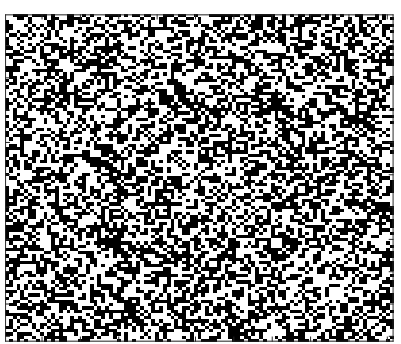

a b

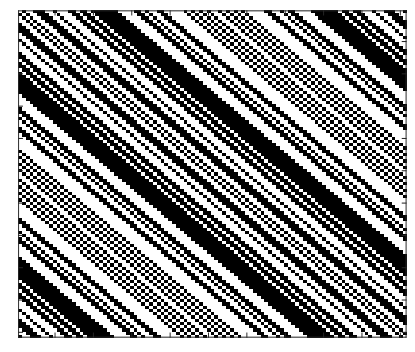

Figure 5: Pixel pictures of Erdős-Rényi (a) and Paley (b) graphs.

\section{Results}

In this section, we present numerical results elucidating some of the implications of the bifurcation analysis outlined in the previous section.

\subsection{Graphs approximating the complete graph}

Our first set of examples deals with the KM on Erdős-Rényi and Paley graphs (see Figure 5). The former is a random graph, whose edges are selected at random from the set of all pairs of vertices with fixed probability $p$ (see Example 2.1). Before giving the definition of the Paley graphs, we first recall the definition of a Caley graph on an additive cyclic group $\mathbb{Z}_{n}=\mathbb{Z} / n \mathbb{Z}$.

Definition 4.1. Let $S$ be a symmetric subset of $\mathbb{Z}_{n}$ (i.e., $S=-S$ ). Then $\Gamma_{n}=\left\langle V\left(\Gamma_{n}\right), E\left(\Gamma_{n}\right)\right\rangle$ is a Caley graph if $V\left(\Gamma_{n}\right)=\mathbb{Z}_{n}$ and $\{a, b\} \in E\left(\Gamma_{n}\right)$, if $b-a \in S$. Caley graph $\Gamma_{n}$ is denoted Cay $\left(\mathbb{Z}_{n}, S\right)$.

Definition 4.2. Let $n=1(\bmod 4)$ be a prime and denote

$$
Q_{n}=\left\{x^{2} \quad(\bmod n): x \in \mathbb{Z}_{n} /\{0\}\right\} .
$$

$Q_{n}$ is viewed as a set (not multiset), i.e., each element has multiplicity 1 . Then $Q_{n}$ is a symmetric subset of $\mathbb{Z}_{n}^{\times}$and $\left|Q_{n}\right|=2^{-1}(n-1)$ (cf. [10. Lemma 7.22]). $P_{n}=\operatorname{Cay}\left(\mathbb{Z}_{n}, Q_{n}\right)$ is called a Paley graph [10].

As all Caley graphs, Paley graphs are highly symmetric (Figure $5 \mathrm{~b}$ ). However, Paley graphs also belong to pseudorandom graphs, which share many asymptotic properties with Erdős-Rényi graphs with $p=1 / 2$ [1]. In particular, both Erdôs-Rényi and Paley graphs have constant graph limit $W_{1 / 2} \equiv 1 / 2$ as $n \rightarrow \infty$. The mean field limit for the KM for Erdôs-Rényi and Paley graphs have the same kernel constant kernel $W=W_{1 / 2} \equiv 1 / 2$ (cf. (3.1)), exactly as in the case of the KM on weighted complete graphs. Since $\mu_{\max }\left(W_{1 / 2}\right)=1 / 2$, the transition to synchronization takes place at

$$
K_{c}^{+}=\frac{4}{\pi g(0)} \approx 3.183
$$

for all three networks (see Figure 6). Here and in the numerical experiments below, $g(x)=(2 \pi)^{-1 / 2} e^{-x^{2} / 2}$. 

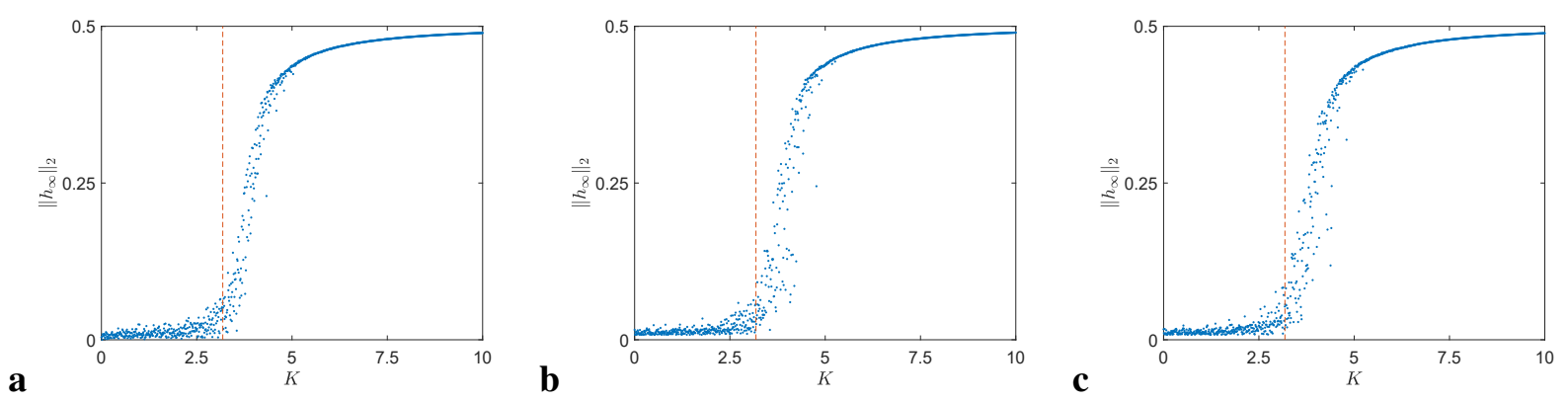

Figure 6: A large time asymptotic value of the order parameter (3.7) (in a scaled $l^{2}$-norm) of is plotted for the KM on complete (a), Erdős-Rényi (b), Paley (c). The transition from 0 to $1 / 2$ takes place in the same region of $K$ in all three plots. The critical value of the coupling strength $K_{c}^{+} \approx 3.2$ is the same for all three models.

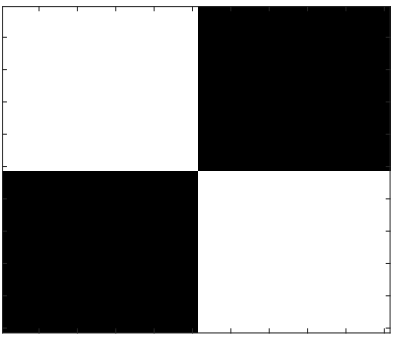

$\mathbf{a}$

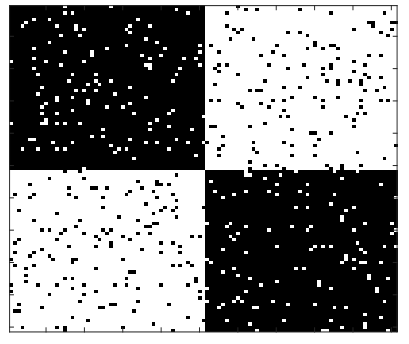

b

Figure 7: Pixel pictures of the bipartite graph (a) and stochastic block graph with two weakly connected components (b). graphs.

\subsection{Bipartite and disconnected graphs}

Our second set of examples features another pair distinct networks, which are not pseudorandom, and yet have the same synchronization threshold.

Here, we consider the KM on bipartite graphs $B_{n, n}=H\left(2 n, W_{b p}\right)$ (see Figure 7(a))

$$
W_{b p}(x, y)= \begin{cases}1, & {[0,0.5) \times[0.5,1] \cup[0.5,1] \times[0,0.5),} \\ 0, & \text { otherwise. }\end{cases}
$$

Next, we consider the family of graphs interpolating between the disconnected graph with two equal components and a weighted complete graphs, $\Gamma_{n, \alpha}=H_{r}\left(n, W_{d, \alpha}\right)$ :

$$
W_{d, \alpha}(x, y)= \begin{cases}1-\alpha, & {[0,0.5) \times[0,0.5) \cup[0.5,1] \times[0.5,1], \quad \alpha \in[0,0.5] .} \\ \alpha, & \text { otherwise }\end{cases}
$$

A simple calculation yields largest positive eigenvalues for each of these families of graphs

$$
\mu_{\max }\left(W_{b p}\right)=\mu_{\max }\left(W_{d, \alpha}\right)=0.5 \quad \forall \alpha \in[0,0.5] .
$$



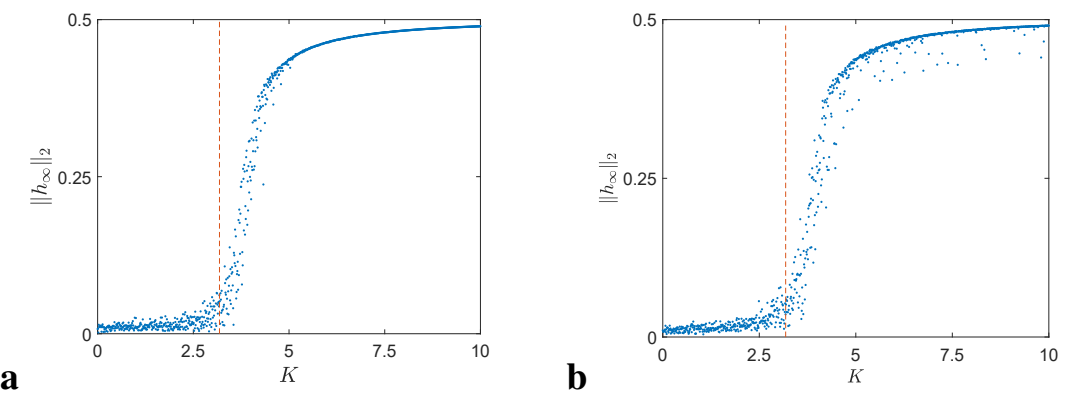

Figure 8: The onset of synchronization in the KM on bipartite graph (a), and on the stochastic block graph with two weakly connected components (b) with $\alpha=.05$.

Thus, as in the first set of examples the $\mathrm{KM}$ on $B_{n, n}$ and $\Gamma_{n, \alpha}$ undergoes transition to synchronization at $K_{c}^{+}=4(\pi g(0))^{-1}$ (see Figure 8 .

\subsection{Power law graphs}

Next we turn to the KM on power law graphs. To generate graphs with power law degree distribution, we use the method of sparse W-random graphs [2]. Specifically, $\Gamma_{n}=H_{r}\left(n, W^{\gamma}, n^{-\beta}\right)$ with graphon $W^{\gamma}$ defined in (2.7). For graphs constructed using this method, it is known that the expected degree of node $i \in[n]$ is $O\left(n^{1+\gamma-\beta}\right)$ and the edge density is $O\left(n^{-\beta}\right)$ (cf. [9]). Thus, $\Gamma_{n}=H_{r}\left(n, W^{\gamma}, n^{-\beta}\right)$ is a family of sparse graphs with power law degree distribution. The mean field limit for the $\mathrm{KM}$ on $\Gamma_{n}$ is given by (3.1) with $W:=W^{\gamma}$. The analysis of the integral operator $\mathbf{W}$ (cf. (3.6) with kernel $W:=W^{\gamma}$ shows that it has a single nonzero eigenvalue (cf. [13])

$$
\mu_{\max }=(1-2 \gamma)^{-1}, \quad \gamma \in(0,0.5) .
$$

Thus, the synchronization threshold for the KM on power law graphs is

$$
K_{c}^{+}=\frac{2(1-2 \gamma)}{\pi g(0)}
$$

Note that as $\gamma \rightarrow 1 / 2, K_{c}^{+}$tends to 0 . Thus, the KM on power law graphs features remarkable synchronizability despite sparse connectivity. Numerics in Figure 9 illustrate the onset of synchronization in power law networks.

\section{Small-world graphs}

Small-world graphs are obtained by random rewiring of regular $k$-nearest-neighbor networks [20]. Consider a graph on $n$ nodes arranged around a circle, with each node connected to $k=\lfloor r n\rfloor$ neighbors from each side for some $r \in(0,0.5)$. With probability $p \in[0,0.5]$ each of these edges connecting $k$ neighbors from 

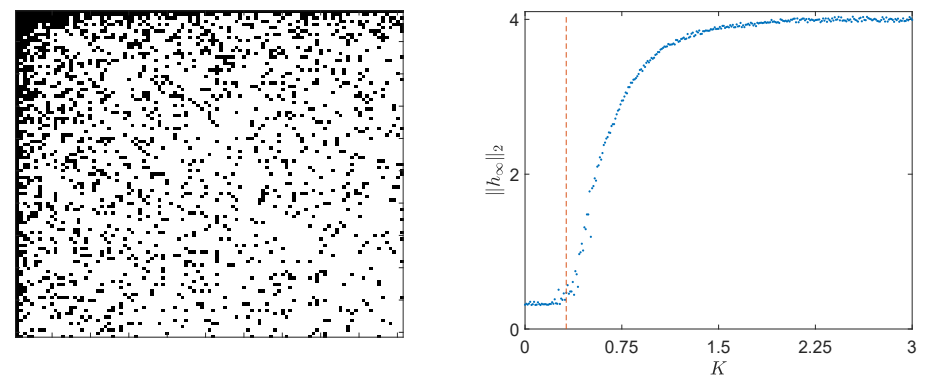

Figure 9: The onset of synchronization in the KM on power-law graphs. The parameter values $\gamma=.4$ and $\beta=.6$ were used in these numerical experiments.

each side, is then replaced by a random long-range (i.e., going outside the $k$-neighborhood) edge. The pixel picture of a representative small-world graph is shown in Figure $2 \mathbf{a}$. A family of small-world graphs parametrized by $p$ interpolates between regular $k$-nearest-neighbor graph $(p=0)$ and a fully random ErdősRényi graph $(p=0.5)$. For intermediate values of $p$, small-world graphs combine features of regular and random connectivity, just as seen in many real-world networks [20].

It is convenient to interpret a small-world graphs as a $W$-random graph $S_{n, p, r}=H_{r}\left(n, W_{r, p}\right)$ (cf. (2.6)). The mean field limit of the KM on $S_{n, p, r}$ is then given by (3.1) with $W:=W_{p, r}$. The corresponding kernel operator is given by

$$
\mathbf{W}_{p, r}(f)=\int_{\mathbb{T}_{1}} K_{p, r}(\cdot-y) f(y) d y, \quad \mathbb{T}_{1}:=\mathbb{R} / \mathbb{Z} .
$$

where $K_{p, r}(x)=(1-p) \mathbf{1}_{|x| \leq r}(x)+p \mathbf{1}_{|x|>r}(x)$.

Using [5.1, we recast the eigenvalue problem for $\mathbf{W}_{p, r}$ as follows

$$
K_{p, r} * v=\mu v .
$$

By taking the Fourier transform of the both sides of $\left[5.2\right.$, we find the eigenvalues of $\mathbf{W}_{p, r}$

$$
\mu_{k}=\int_{\mathbb{T}} K_{p, r}(x) e^{-2 \pi i k x} d x= \begin{cases}(2 r+p-4 r p), & k=0, \\ (\pi k)^{-1}(1-2 p) \sin (2 \pi k r), & k \in \mathbb{Z} /\{0\} .\end{cases}
$$

The corresponding eigenvectors are $w_{k}=e^{i 2 \pi k x}, k \in \mathbb{Z}$. Note that $\mu_{k}=\mu_{-k}$, since $K_{p, r}$ is even (cf. (5.3)). Thus, the eigenspace corresponding to $\mu=\mu_{0}$ is spanned by $w_{0}=1$. For $\mu \neq \mu_{0}$, the eigenspace corresponding to $\mu$ is spanned by

$$
w_{k}=e^{i 2 \pi k x} \quad \text { and } \quad w_{-k}=e^{-i 2 \pi k x}, \quad k \in I_{\mu},
$$

where $I_{\mu}=\left\{k \in \mathbb{N}: \mu_{k}=\mu\right\}$. The largest positive eigenvalue of $\mathbf{W}_{p, r}$ is

$$
\mu_{0}=\int_{I} K_{p, r}(x) d x=2 r+p-4 p r .
$$


Therefore, the onset of synchronization in the KM on small-world graphs takes place at

$$
K_{c}^{+}=\frac{2}{\pi g(0)(2 r+p-4 p r)}
$$

(see Figure 33).

Importantly, $\mathbf{W}_{p, r}$ also has negative eigenvalues. Since $\mathbf{W}_{p, r}$ is a compact operator, 0 is the only accumulation point of the spectrum of $\mathbf{W}_{p, r}$. Thus, there is the smallest (negative) eigenvalue $\mu_{\min }=\min \left\{\mu_{k}\right.$ : $k \in \mathbb{N}\}$. Let $q \in \mathbb{N}$ be such that $\mu_{q}=\mu_{\text {min }}$. Assuming that the multiplicity of $\mu_{\text {min }}$ is 2 , the center manifold reduction performed for the order parameter in [5] yields the following stable branch bifurcating from the incoherent state $(h \equiv 0)$ at $K=K_{c}^{-}$

$$
h_{\infty}(x, K)=\sqrt{\frac{\kappa}{\beta}} e^{ \pm i 2 \pi q(x+\phi)}+o(\sqrt{\kappa}), \quad 0<\kappa:=K_{c}^{-}-K \ll 1, \beta:=-\frac{\pi g^{\prime \prime}(0)\left(K_{c}^{-}\right)^{4} \mu_{\min }}{16},
$$

where $\phi \in[0,1)$ depends on the initial data.

Equation (5.5) implies that at $K=K_{c}^{-}$the KM on small-world graphs undergoes a pitchfork bifurcation. Unlike the bifurcation at $K=K_{c}^{+}$considered earlier, in the present case the center manifold is twodimensional. It is spanned by $w_{ \pm q}=e^{ \pm i 2 \pi q x}$. In the remainder of this section, we analyze stable spatial patterns bifurcating from the incoherent state at $K=K_{c}^{-}$. To this end, we rewrite the velocity field (3.3), using the order parameter:

$$
V(t, u, \omega, x)=\omega+\frac{K}{2 i}\left(e^{-i u} h(t, x)-e^{i u} \overline{h(t, x)}\right) .
$$

The velocity field in the stationary regime is then given by

$$
V_{\infty}(u, \omega, x)=\omega+\frac{K}{2 i}\left(e^{-i u} h_{\infty}(x, K)-e^{i u} \overline{h_{\infty}(x, K)}\right) .
$$

Using the polar form of the order parameter

$$
h_{\infty}\left(x, K_{c}^{-}-\kappa\right)=R_{\infty}(x, K) e^{i \Phi(x)}, \quad R_{\infty}(x, K)=\sqrt{\frac{\kappa}{\beta}}+o(\sqrt{\kappa}), \Phi(x)= \pm 2 \pi q(x+\phi),
$$

from (5.7) we obtain

$$
V_{\infty}(u, \omega, x)=\omega-\left(K_{c}^{-}-\kappa\right) \sqrt{\frac{\kappa}{\beta}} \sin (u-\Phi(x))+o(\sqrt{\kappa})
$$

Since $\rho_{\infty}$ is a steady state solution of the (3.1), it satisfies

$$
\partial_{u}\left\{V_{\infty} \rho_{\infty}\right\}=0
$$

From 5.9 and 5.10, we have

$$
\rho_{\infty}(u, \omega, x)= \begin{cases}\delta\left(u-\Phi(x)-\arcsin \left(\omega \frac{\sqrt{\beta}}{K \sqrt{\kappa}}\right)+o(\sqrt{\kappa})\right), & |\omega| \leq K R_{\infty}(x, K), \\ \frac{1}{2 \pi} \frac{\sqrt{\omega^{2}-K^{2} R_{\infty}(x, K)^{2}}}{\omega-K R_{\infty}(x, K) \sin (u-\Phi(x)) \mid}, & |\omega|>K R_{\infty}(x, K) .\end{cases}
$$



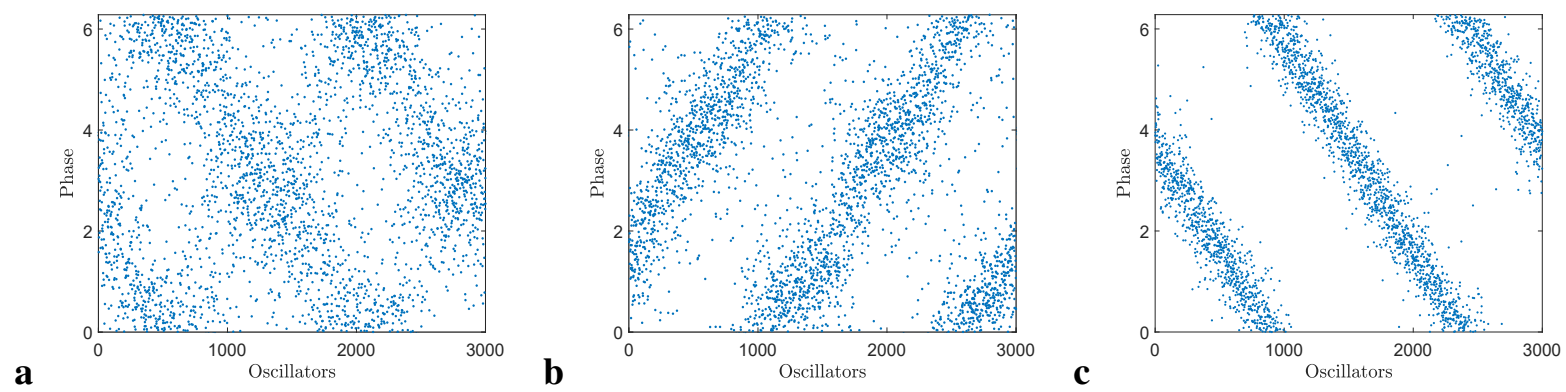

Figure 10: The formation of 2-twisted states for decreasing values of coupling strength $K$. From a to $\mathbf{c}$, we have, respectively, $K=-32, K=-36$, and $K=-50$. In all simulations $p=.2$ and $r=.3$.

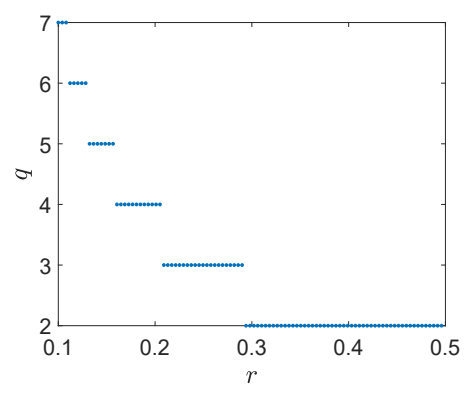

Figure 11: The winding number $q$ of the twisted states bifurcating from the incoherent state as a function of the connectivity range $r$.

Here, $K=K_{c}^{-}-\kappa$ and $\delta$ stands for the Dirac delta function. Equation (5.11) describes partially phase locked solutions. They combine phase locked oscillators (type I)

$$
u= \pm 2 \pi q(x+\phi)+Y(\omega, \kappa)+O(\sqrt{\kappa}), \quad Y(\omega, \kappa)=\arcsin \left(\omega \frac{\sqrt{\beta}}{K \sqrt{\kappa}}\right)
$$

and those whose distribution is given by the second line in (5.11) (type II). The oscillators of the first type form $q$-twisted states subject to noise $Y$. Note that $Y$ is a function of $\omega$ and, therefore, is random. Just near the bifurcation, where $0<\kappa \ll 1$, the probability of $|\omega|>K R_{\infty}(x, K)$ is high and, thus, most oscillators are of the second type (Figure 10a). However, as we move away from the bifurcations, we see the number of the oscillators of the first type increase and the noisy twisted states become progressively more distinct (Figure $10 \mathbf{b}, \mathbf{c}$ ). Thus, the bifurcation analysis of the KM on small-world graphs identifies a family of stable twisted states (cf. [17, 21]). By changing parameters of small-world connectivity, one can control the winding number of the emerging twisted states (see Figure 11). 


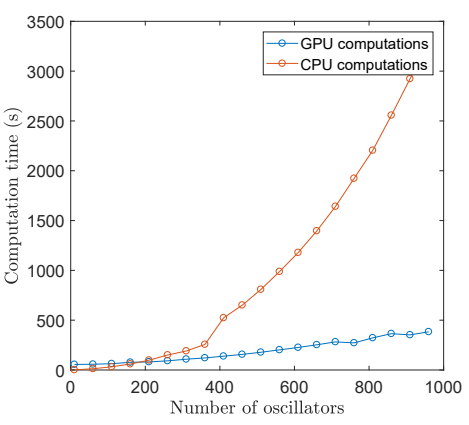

Figure 12: Comparison of GPU and CPU computation time on Intel(R) Core i7-7700 CPU with NVIDIA Quadro K1200 GPU showing dramatic speed-up due to parallelization of computations.

\section{Discussion}

In this paper, we selected several representative families of graphs to illustrate the link between network topology and synchronization and pattern formation in the KM of coupled phase oscillators. In particular, we showed that the transition to synchronization in the KM on pseudorandom graphs (e.g., Erdôs-Rényi, Paley, complete graphs) starts at the same critical value of the coupling strength and proceeds in practically the same way. The bifurcation plots shown in Figure 6 are very similar, although plots for the KM on ErdősRényi and Paley graphs show more variability. The almost identical bifurcation plots for these models are due to the fact that all three models result in the same mean field equation. This means that the empirical measures generated by the trajectories of these models are asymptotically have the same limit as $n \rightarrow \infty$. The differences seen in plots a-c of Figure 6 are due to the higher order moments, which are not captured by the mean field limit. Other families of graphs considered in this paper include the bipartite graph and a family of stochastic block graphs interpolating between a graph with two disconnected components and ErdősRényi graph. The KM on all these graphs (including the disconnected graph) feature the same transition to synchronization. Finally, we studied the bifurcations in the KM on small-world and power law graphs due to their importance in applications. A remarkable feature of the KM on small-world graphs is the presence of the bifurcation leading to stable noisy twisted states. Twisted states were known as attractors in repulsively coupled KM with identical intrinsic frequencies [21, 16, 17, 18]. In this paper, we show that twisted states (albeit noisy) can also be attractors in the KM with random intrinsic frequencies. Furthermore, they bifurcate from the incoherent, i.e. fully random, steady state.

To perform numerical experiments presented in this paper we had to overcome several challenges. Verification of the bifurcation scenarios in the KM required a large number of simulations of large systems of ordinary differential equations with random coefficients. Thus, the speed of computations was critical in this project. All computations were completed in MATLAB utilizing GPU computations for dramatic speed-up in the computational time (compared to CPU computations), see Figure 12 . Time steps were performed using Heun's method with $\Delta t=.01$, which was sufficient for stable simulation results.

Each simulation was initialized with a random state vector $\left(u_{n, 1}, \ldots, u_{n, n}\right)^{T}$ with each component independently chosen from a uniform distribution on $[0,2 \pi)$ representing the phase of each oscillator. We 
additionally initialize a random vector of internal frequencies chosen from a normal distribution. Unless otherwise noted, in all simulations we take $n=4001$, which is a prime 1 modulo 4 , so that the theory developed for Paley graphs applies. We observed that taking $T_{\text {final }}=20$ was sufficient for systems to exhibit synchronization or $q$-twisted states.

Computationally solving (5.3) for the minimal negative eigenvalue is accomplished by observing the trivial bound $\mu_{k}>\frac{-1}{k \pi}$ and iteratively computing $\mu_{\text {min }}=\mu_{k_{0}}$ for $k_{0} \in\{1, \ldots, M\}$ for increasing values of $M$ until $\mu_{\min } \leq \frac{-1}{M \pi}$.

\section{Acknowledgments}

The work of the second author was supported in part by the NSF DMS 1715161. The authors would like to thank Nicholas Battista for helpful discussions related to implementation of the numerical simulations. 


\section{References}

[1] N. Alon and J. H. Spencer, The probabilistic method, fourth ed., Wiley Series in Discrete Mathematics and Optimization, John Wiley \& Sons, Inc., Hoboken, NJ, 2016.

[2] C. Borgs, J. T. Chayes, H. Cohn, and Y. Zhao, An $L^{p}$ theory of sparse graph convergence I: limits, sparse random graph models, and power law distributions, ArXiv e-prints (2014).

[3] H. Chiba, A proof of the Kuramoto conjecture for a bifurcation structure of the infinite-dimensional Kuramoto model, Ergodic Theory Dynam. Systems 35 (2015), no. 3, 762-834.

[4] H. Chiba and G. S. Medvedev, The mean field analysis of the Kuramoto model on graphs I. The mean field equation and the transition point formulas, submitted.

[5] _ The mean field analysis of the Kuramoto model on graphs II. Asymptotic stability of the incoherent state, center manifold reduction, and bifurcations, submitted.

[6] H. Dietert, Stability and bifurcation for the Kuramoto model, J. Math. Pures Appl. (9) 105 (2016), no. 4, 451-489.

[7] B. Fernandez, David Gérard-Varet, and G. Giacomin, Landau damping in the Kuramoto model, Ann. Henri Poincaré 17 (2016), no. 7, 1793-1823.

[8] D. Kaliuzhnyi-Verbovetskyi and G. S. Medvedev, The mean field equation for the kuramoto model on graph sequences with non-Lipschitz limit, SIAM J. Math. Anal. in press.

[9] _ The semilinear heat equation on sparse random graphs, SIAM J. Math. Anal. 49 (2017), no. 2, 1333-1355.

[10] M. Krebs and A. Shaheen, Expander families and Cayley graphs, Oxford University Press, Oxford, 2011, A beginner's guide.

[11] L. Lovász, Large networks and graph limits, AMS, Providence, RI, 2012.

[12] L. Lovász and B. Szegedy, Limits of dense graph sequences, J. Combin. Theory Ser. B 96 (2006), no. 6, 933-957.

[13] G. S. Medvedev and X. Tang, The Kuramoto model on power law graphs, ArXiv e-prints (2017).

[14] G. S. Medvedev, The nonlinear heat equation on dense graphs and graph limits, SIAM J. Math. Anal. 46 (2014), no. 4, 2743-2766.

[15] _ The nonlinear heat equation on W-random graphs, Arch. Ration. Mech. Anal. 212 (2014), no. $3,781-803$.

[16]__ Small-world networks of Kuramoto oscillators, Phys. D 266 (2014), 13-22.

[17] G. S. Medvedev and X. Tang, Stability of twisted states in the Kuramoto model on Cayley and random graphs, Journal of Nonlinear Science (2015). 
[18] G. S. Medvedev and J. Wright, Stability of twisted states in the continuum Kuramoto model, SIAM J. Appl. Dyn. Syst. 16 (2017), no. 1, 188-203.

[19] S. H. Strogatz, From Kuramoto to Crawford: exploring the onset of synchronization in populations of coupled oscillators, Phys. D 143 (2000), no. 1-4, 1-20, Bifurcations, patterns and symmetry.

[20] D. J. Watts and S. H. Strogatz, Collective dynamics of small-world networks, Nature 393 (1998), 440442.

[21] D. A. Wiley, S. H. Strogatz, and M. Girvan, The size of the sync basin, Chaos 16 (2006), no. 1, 015103, 8. 